OPEN ACCESS

Edited by:

Eugenia Bezirtzoglou,

Democritus University of Thrace,

Greece

Reviewed by:

Birce Mercanoglu Taban,

Ankara University, Turkey

Yiannis Kourkoutas,

Democritus University of Thrace,

Greece

*Correspondence:

Aspasia Nisiotou

anisiotou.wi@nagref.gr

Specialty section:

This article was submitted to

Food Microbiology,

a section of the journal

Frontiers in Microbiology

Received: 04 May 2021

Accepted: 24 May 2021

Published: 30 June 2021

Citation:

Chalvantzi I, Banilas G, Tassou C and

Nisiotou A (2021) Biogeographical

Regionalization of Wine Yeast

Communities in Greece and

Environmental Drivers of Species

Distribution at a Local Scale.

Front. Microbiol. 12:705001.

doi: 10.3389/fmicb.2021.705001

\section{Biogeographical Regionalization of Wine Yeast Communities in Greece and Environmental Drivers of Species Distribution at a Local Scale}

\author{
loanna Chalvantzi ${ }^{1,2}$, Georgios Banilas ${ }^{2}$, Chrysoula Tassou ${ }^{1}$ and Aspasia Nisiotou ${ }^{1 *}$ \\ ${ }^{1}$ Hellenic Agricultural Organization "Dimitra", Institute of Technology of Agricultural Products, Lykovrysi, Greece, \\ ${ }^{2}$ Department of Wine, Vine and Beverage Sciences, University of West Attica, Athens, Greece
}

Recent research has expanded our understanding on vineyard-associated fungal community assembly, suggesting non-random distribution and implicating regional differences in the wine terroir effect. Here, we focused on the culturable fraction of the fungal community that resides on grapes and determine wine quality, the so-called wine yeast populations. We aimed to analyze local-scale yeast community assemblages and to test whether the hypothesis of biogeographical patterns also applies to wine yeasts in particular. Surveying 34 vineyards across four main viticultural zones in Greece showed significant trends in vineyard-specific patterns. At a local scale, viticultural regions were also linked to distinct yeast community compositions. Importantly, major yeast populations directly related to wine fermentation contributed significantly to the delimitation of regions, highlighting their potential influence on the regionality of wine characteristics. In terms of the microbial terroir influence, yeast communities within an area were temporarily stable, which is critical for the regional character of the wine. Community structure could be explained only partially by environmental features. Maximum temperature, elevation, and net precipitation were the highest correlated variables with the yeast community biogeographic patterns. Finally, we also showed that certain environmental factors may drive the population size of specific yeast populations. The present results indicate that the wine yeast community has a geographical character at local scale, which is an important feature of the microbial terroir concept and thus for the wine industry.

Keywords: yeasts, microbial diversity, microbial biogeography, microbial terroir, wine

\section{INTRODUCTION}

The grape-associated yeast community is a significant component of the vine-wine system and is vastly associated with the quality of wine. Yeast community builds up concomitantly with grape berry development reaching densities of 3-5 Log CFU/g in ripe healthy grapes that may increase up to $7 \mathrm{Log} \mathrm{CFU} / \mathrm{g}$ on damaged or botrytised grapes (Nisiotou and Nychas, 2007). Yeast species richness and relative species abundance are also in line with the stage of grape maturation. Grapes soon after the onset of ripening (veraison) are mainly colonized by basidiomycetous yeasts, such as Cryptococcus spp., Rhodotorula spp., Sporobolomyces spp., and 
the yeast-like fungus Aureobasidium pullulans, while thereafter they are gradually occupied by oxidative or weakly fermentative ascomycetous species, such as Hanseniaspora spp., Metschnikowia spp., Pichia spp., and Candida spp. (Barata et al., 2012). According to multiple reports, Hanseniaspora uvarum and Metschnikowia pulcherrima are the dominant species on mature healthy grapes, especially in warm climate regions (Nisiotou and Nychas, 2007; Settanni et al., 2012; Drumonde-Neves et al., 2017). To a smaller extent, it is possible to detect Candida, Cryptococcus, Lachancea, Rhodotorula, Pichia, and Torulaspora species, while Saccharomyces cerevisiae, the main wine fermenting yeast, is very scarce (Barata et al., 2012; Drumonde-Neves et al., 2017). Overripe, damaged, or botrytised grape berries support the growth of yeasts with high fermentative power and also of spoilages species like Pichia spp., Zygoascus hellenicus, Zygosaccharomyces spp., and Torulaspora spp. (Nisiotou et al., 2007; Nisiotou and Nychas, 2007; Barata et al., 2012).

The composition of grape microbial communities is affected by a number of factors including the sanitary state of grape berries, the climatic conditions, and the farming system (Nisiotou et al., 2007; Chavan et al., 2009; Li et al., 2010; Cordero-Bueso et al., 2011; Drumonde-Neves et al., 2017). Geographic separation has been suggested as another factor that may shape fungal grape microbial communities (Bokulich et al., 2014). Contrary to the Baas Becking hypothesis as "everything is everywhere but the environment selects," it seems that the dispersal limitation may affect microbial communities. Recent data suggest a negative correlation between similarity of microbial communities and geographic distance, irrespective of the environmental factors, supporting thereby the existence of geospatial structures for microbial assemblages (Peay et al., 2010; Finkel et al., 2012; Cline and Zak, 2014; Morrison-Whittle and Goddard, 2015). Miura et al. (2017) showed that grape fungal community differences increased with geographic distance introducing thereby spatial processes as important factors in shaping yeast biogeographic patterns of vineyards.

Geographical patterns of grape-associated yeast communities form the basis for introducing a microbial notion to the terroir concept. Terroir, which expresses the influence of the place of origin in the special features of a food product, affects significantly consumer preferences and economic appreciation of wines (Van Leeuwen and Seguin, 2006). Still, the drivers and dimensions of wine "microbial terroir" have not been described in a comprehensive way. A growing body of research though indicates that wine yeast community patterns may correlate with the region of origin. For instance, geographical delineations of yeast communities have been documented in New Zealand vineyards (Gayevskiy and Goddard, 2012). Regional factors were also found to affect the fungal biodiversity within Californian sub-regions (Bokulich et al., 2014) or Portuguese appellations (Pinto et al., 2015). Similarly, Drumonde-Neves et al. (2017) observed significant differences among yeast communities in vineyards of five islands of the Azores Archipelago. Using next-generation sequencing (NGS) technology and metagenomics approaches, Taylor et al. (2014) and Morrison-Whittle and Goddard (2018) also detected significant differences in grape and must fungal communities at the genus level of different regions in New Zealand. Despite the advantages, due to the existence of numerous other fungi in the sample, NGS may provide proportionally more information on non-enological yeasts and fungi rather than to $S$. cerevisiae and non-Saccharomyces wine yeasts (Alexandre, 2020). However, these species contribute the most to wine fermentation and ultimately shape the wine phenotype and quality. Regional differences in yeast communities were further correlated to distinct wine metabolomes. Grape microbiota and wine metabolite profiles could distinguish viticultural area designations and individual vineyards within Napa and Sonoma Counties in California (Bokulich et al., 2016). Knight et al. (2015) showed that genetically differentiated regional populations of $S$. cerevisiae in New Zealand differentially affect wine phenotype. A positive correlation was also observed between phenotypic and genotypic diversity of regionally differentiated Lachancea thermotolerans vineyard strains in Greece (Banilas et al., 2016) as the first indication that the microbial terroir concept may also apply to strains of non-Saccharomyces wine yeast species.

Many questions about vineyard microbiota and their dispersion among vineyards and regions remain unanswered (Griggs et al., 2021). Here, we followed a culture-depended approach to isolate the grape-associated wine yeasts and to compare the yeast species assemblages within and between four major wine-producing regions in Greece (Peza in Crete, Santorini island, and the ancient viticultural zones of Nemea and Mantineia) over two consecutive years. The above regions form unique viticultural ecosystems as defined by inherent climatic and topographical features and historical viticultural practices. In the present study, the structure of wine yeast communities as a part of these peculiar ecosystems was questioned and potential drivers of regional yeast community assembly were investigated as per their role in the configuration of vine-wine microbial systems at local scale.

\section{MATERIALS AND METHODS}

\section{Grape Sampling}

Grape samples were collected from the following Protected Designation of Origin viticultural regions across southern Greece on an N-S maximum distance of $\sim 370 \mathrm{~km}$ : Nemea in Peloponnese peninsula (11 vineyards, intermediate temperature zone), Mantineia plateau in Peloponnese peninsula (two vineyards, cool temperature zone), Santorini island (six vineyards in vintages $\mathrm{A}$ and $\mathrm{B}$ and three vineyards in vintage $\mathrm{C}$; warm zone), and Peza in Crete island (10 vineyards in vintage A and four vineyards in vintage B; warm zone; Figure 1). Regions were sampled based on their size and their relative spatial heterogeneity. According to the National Inter-professional Organization of Vine and Wine portal for Greece's vineyards and wines, ${ }^{1}$ Nemea and Peza are the largest viticultural regions (30 $\mathrm{km}^{2}$ cultivated vineyards each) and present the most heterogeneous landscapes among the areas surveyed. Nemea is divided into three altitude zones, the lowland (at elevation of $260-350 \mathrm{~m}$ ), the semi-mountainous $(350-600 \mathrm{~m})$, and the

\footnotetext{
${ }^{1}$ https://winesofgreece.org
} 


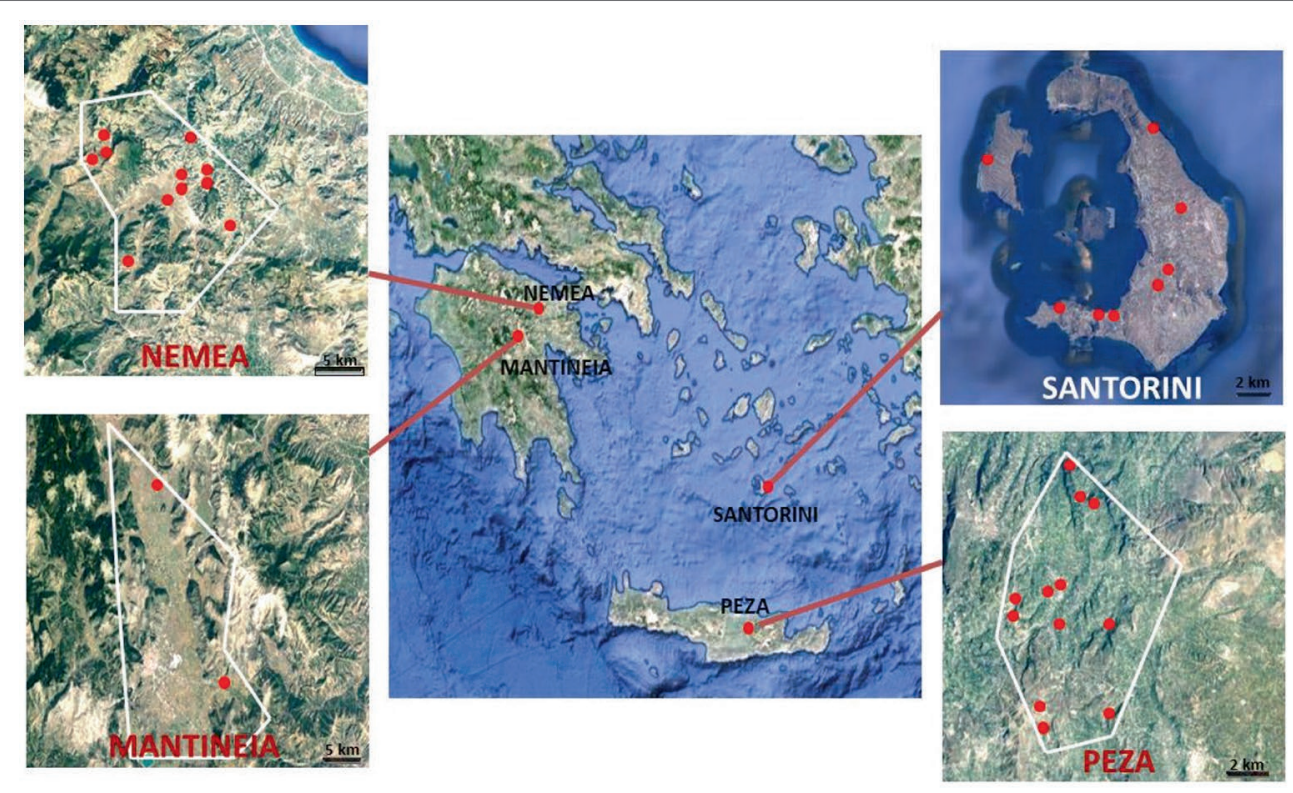

FIGURE 1 | Maps of the vineyards sampled in four major PDO regions in Greece (Nemea and Mantineia in Peloponnese, Santorini island, and Peza in Crete). Images taken from Google Maps (c) 2021 TerraMetrics, Map data (c) 2021 Google.

mountainous (600-800 m). In Peza, the vines are spread amphitheatrically on low hills with varying slope up to an altitude of $700 \mathrm{~m}$ (mountainous and semi-mountainous vineyards). Santorini has $15 \mathrm{~km}^{2}$ of vineyards, located in the central and the southern parts of the island. Mantineia is the smallest viticultural region $\left(10 \mathrm{~km}^{2}\right)$, which presents a uniform landscape, located on a mountainous plateau at an altitude of $650 \mathrm{~m}$. Two to nine sampling plots per vineyard were surveyed as previously described (Chalvantzi et al., 2020) giving a total of 156 grape samples. Grapes were placed into sterile plastic bags, transferred to the laboratory and crushed with a stomacher.

\section{Analytical Determinations}

Reducing sugars, total acidity, and $\mathrm{pH}$ determinations were estimated by the methods described in the "Compendium of International Methods of Analysis of Musts and Wines," International Organization of Vine and Wine (OIV, 2015).

\section{Isolation and Identification of Yeasts}

For the enumeration and isolation of yeasts, grape juice serial dilutions were plated into Wallerstein laboratory nutrient agar, lysine medium agar, and ethanol sulfite agar supplemented with $100 \mathrm{mg} / \mathrm{l}$ biphenyl and $100 \mathrm{mg} / \mathrm{l}$ chloramphenicol. Colonies were randomly selected from plates, purified by streak plating technique and examined microscopically. Isolates were stored at $-80^{\circ} \mathrm{C}$ until further analysis. Cells were collected from plates with a sterile yellow tip and suspended in $3 \mu \mathrm{l}$ of $\mathrm{NaOH} 0.02 \mathrm{M}$ solution. Cell suspensions were then incubated at $99^{\circ} \mathrm{C}$ for $10 \mathrm{~min}$. The ITS-5.8S rDNA region was PCR-amplified using the primers ITS1 and ITS4 (White et al., 1990). Sizes of amplicons were determined using a 100-bp molecular weight marker in $1.2 \%(\mathrm{w} / \mathrm{v})$ agarose gels. For species-level assignment $500 \mathrm{ng}$ of PCR products were digested by Hinfl, HaeIII, or HhaI according to Esteve-Zarzoso et al. (1999) and analyzed on 3\% (w/v) agarose gel. Further digestions by the restriction enzymes DdeI and DraI were performed to differentiate between Hanseniaspora and Candida genera as previously suggested (Nisiotou et al., 2007). PCR products of representative yeast isolates per distinct restriction pattern were purified using the QIAquick PCR purification kit and directly sequenced (Macrogen). ${ }^{2}$ BLAST searches of sequences were performed at the NCBI/GenBank database, and the Clustal X software was used to construct multiple sequence alignments.

\section{Climate Data}

Climatic data were collected from weather stations located in the vineyard regions. Daily measurements for average high temperature, average low temperature, average temperature, maximum temperature, minimum temperature, net precipitation, average wind speed, maximum wind speed, and average maximum wind speed were obtained.

\section{Statistical Analysis}

Differences in yeast community composition between regions, vineyards, or vintages were tested with Analysis of Similarity (ANOSIM) on square-root transformed species incidences using the Bray-Curtis distance matrices. ANOSIM R statistic close to the maximum limit of one implies robust group separation rejecting the null hypothesis $R=0$ (no differences between groups), while negative values of $R$ show higher intra-group than inter-group diversity. Values of $p<0.05$ indicate significantly different level between groups. When the null hypothesis was rejected, pairwise values of $R$ were

${ }^{2}$ http://www.macrogen.com 
examined to reveal major differences between regions. Permutational multivariate analysis of variance (PERMANOVA) tests with 999 permutations was also applied to test significant differences between sample groups using Bray-Curtis similarity measure, where higher values of $F$ statistic indicate greater between-groups variations. Cluster analysis dendrograms were constructed based on Bray-Curtis similarity matrices to test clustering of yeast communities according to the vineyard or the region of origin. Principal coordinate analysis (PCoA) based on Bray-Curtis distances was applied, as an unconstrained method, to depict resemblances of samples in a two-dimensional space. Following PCoA, canonical discriminant analysis (CDA), a constrained ordination method where the groups are defined a priori, was used to produce an ordination plot and compare samples of different regions against species abundances, according to Anderson and Willis (2003). Ordination by non-metric multidimensional scaling (nMDS) was also used to reflect yeast community resemblances by projecting the pairwise dissimilarity between objects in a two-dimensional space as accurately as possible (Legendre and Legendre, 1998; Ramette, 2007). Mantel test (Spearman's rank correlations with 999 permutations), a widely used test to compare matrices calculated for the same objects but from independent data sets (Ramette, 2007), was applied to determine the correlation between geographic distance of regions and yeast community (dis)similarity matrices. Significant differences between environmental conditions of different regions or vintages were tested with ANOVA. BEST was applied as described (Clarke and Gorley, 2015) to identify the most important environmental factors that drive community differences across regions. Distance-based linear model (DistLM) marginal test was applied to evaluate correlation of the environmental factors with the yeast community structure patterns and to test significance. Distance-based redundancy analysis (db-RDA) was used for the ordination and visualization of DistLM (Anderson et al., 2008). Partial least squares regression (PLSR) model was applied to demonstrate associations between environmental variables (predictor variables $\mathrm{X}$ ) and specific yeast populations (response variables $\mathrm{Y}$ ) and to detect particular climatic factors that drive the distribution of yeast populations across regions. All statistical tests were performed using the software PRIMER Version 7 with PERMANOVA $+{ }^{3}$ except for PLSR and CDA that were conducted with JMP. ${ }^{4}$

\section{RESULTS}

\section{Wine Yeast Species Abundance and Community Heterogeneity}

A total of 156 grape samples derived from 34 vineyards covering highly important viticultural regions across Greece were analyzed (Figure 1). Yeast counts differed significantly among regions $(p<0.001)$. Samples from the Santorini island exhibited the highest yeast counts $(5.85 \pm 1.06 \mathrm{Log} \mathrm{CFU} / \mathrm{ml})$ followed by

${ }^{3}$ https://www.primer-e.com

${ }^{4}$ https://www.jmp.com
Mantineia (5.49 \pm 0.30 Log CFU/ml), Peza (4.54 $\pm 0.98 \mathrm{Log}$ $\mathrm{CFU} / \mathrm{ml})$, and Nemea (3.66 $\pm 1.10 \mathrm{Log} \mathrm{CFU} / \mathrm{ml})$. Species richness was also significantly higher $(p<0.05)$ in Santorini (16 species) compared to the other regions.

Yeast isolates were analyzed by PCR-RFLP analysis of the 5.8S-ITS rDNA region. Restriction enzyme banding profile comparisons between isolates and published strains combined with sequence analysis of the 5.8S-ITS region assigned groups of isolates to 18 different yeast species, namely, Aureobasidium pullulans, Candida diversa, C. glabrata, C. membranifaciens, C. tropicalis, Cryptococcus diffluens, $H$. uvarum, H. guilliermondii, H. opuntiae, Hyphopichia pseudoburtonii, Lachancea thermotolerans, Meyerozyma caribbica, M. pulcherrima, Papiliotrema laurentii, Pichia anomala, S. cerevisiae, Starmerella bacillaris, and Torulaspora delbrueckii. Except for P. laurentii, the majority of the species belonged to Ascomycota, including species that significantly contribute to the alcoholic fermentation of grape must. Figure 2 shows the species diversity and their relative abundance in the different viticultural regions studied. M. pulcherrima was the most frequently isolated yeast (Figure 2; $37 \%)$. M. pulcherrima was highly encountered in Peza (70\%), followed by Nemea (50\%). It was moderately abundant in Mantineia (27\%), but scarcely detected in Santorini (1\%). $H$. uvarum was the second most encountered species across vineyards. It was highly abundant in Mantineia (74\%) and moderately found in other regions, i.e., $25 \%$ in Santorini, $17 \%$ in Nemea, and $15 \%$ in Peza.

Only five out of a total 18 species were inter-regionally encountered, i.e., S. bacillaris, $H$. guilliermondii, $H$. uvarum, M. caribbica, and $M$. pulcherrima. Several species were associated exclusively with Santorini, namely, C. diversa, C. glabrata, C. membranifaciens, P. laurentii, C. tropicalis, S. cerevisiae, and T. delbrueckii, while $H$. pseudoburtonii and P. anomala were solely encountered in Peza.

\section{Inter-Regional Yeast Community Similarities}

We further asked whether there is any difference between the yeast communities of different viticultural regions. Both ANOSIM and PERMANOVA tests indicated that species composition of yeast communities differed significantly among regions (ANOSIM: $R=0.401, p=0.001$; Table 1). ANOSIM pairwise tests revealed that all pairs of regions shared significantly dissimilar communities, except for the nearby Mantineia and Nemea regions, both located in Peloponnese, and Mantineia vs. Santorini (Table 2).

A Bray-Curtis similarity dendrogram was constructed (Cophenetic correlation: 0.93) to compare yeast communities of the four regions (Figure 3A). Most samples of the same origin were placed together and rather separated from those of other regions, supporting the results of both ANOSIM and PERMANOVA. Nevertheless, a close relationship was observed for some samples from Nemea and Peza. PCoA based on Bray-Curtis dissimilarities of species composition among the samples depicted clustering of samples from the same region, with Nemean samples being more dispersed 


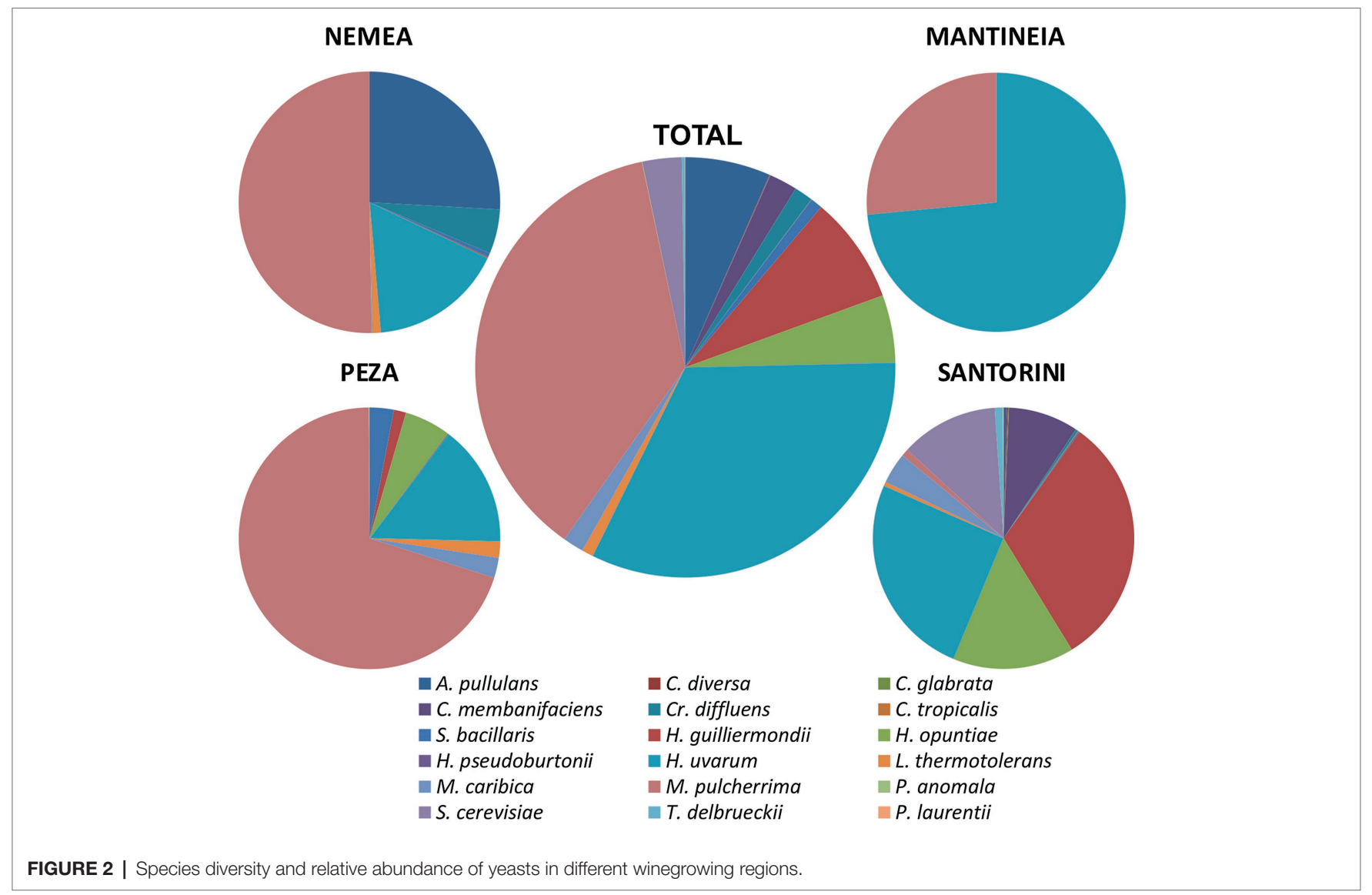

TABLE 1 | Analysis of Similarity (ANOSIM) and Permutational multivariate analysis of variance (PERMANOVA) of the factors "region," "vintage," and "vineyard" on wine yeast diversity patterns.

\begin{tabular}{|c|c|c|c|c|c|c|}
\hline \multirow{2}{*}{ Region } & \multirow{2}{*}{ Vintage } & \multirow{2}{*}{ Factor } & \multicolumn{2}{|c|}{ ANOSIM } & \multicolumn{2}{|c|}{ PERMANOVA } \\
\hline & & & $\boldsymbol{R}$ & $p$ & $\boldsymbol{F}$ & $p$ \\
\hline All & All & Region & 0.401 & $0.001 *$ & 20.339 & $0.001 *$ \\
\hline All & $A$ & Region & 0.525 & $0.001 *$ & 14.678 & $0.001 *$ \\
\hline All & B & Region & 0.480 & $0.001 *$ & 12.207 & $0.001 *$ \\
\hline All & All & Vintage & 0.043 & 0.138 & 3.4479 & $0.014 *$ \\
\hline Nemea & All & Vintage & -0.038 & 0.834 & 0.1001 & 0.908 \\
\hline Peza & All & Vintage & -0.022 & 0.506 & 0.6741 & 0.517 \\
\hline Santorini & All & Vintage & 0.379 & $0.001 *$ & 6.3601 & $0.001 *$ \\
\hline Mantineia & All & Vintage & 0.000 & 0.400 & 0.0758 & 1.000 \\
\hline All & All & Vineyard & 0.508 & $0.001 *$ & 12.525 & $0.001 *$ \\
\hline Nemea all & All & Vineyard & 0.388 & $0.001 *$ & 6.2636 & $0.001 *$ \\
\hline Nemea & $A$ & Vineyard & 0.605 & $0.030 *$ & 8.5558 & $0.005^{*}$ \\
\hline Nemea & B & Vineyard & 0.260 & 0.104 & 3.3663 & $0.042 *$ \\
\hline Santorini & $A, B$ & Vineyard & 0.471 & $0.001 *$ & 12.350 & $0.001 *$ \\
\hline Santorini & A & Vineyard & 0.820 & $0.001 *$ & 13.015 & $0.001 *$ \\
\hline Santorini & $\mathrm{B}$ & Vineyard & 0.456 & $0.044 *$ & 2.3175 & 0.055 \\
\hline Peza & $A$ & Vineyard & 0.121 & 0.110 & 3.1363 & $0.035^{*}$ \\
\hline
\end{tabular}

$* p<0.05$

than others and also related to samples from Peza (Figure 3B). CDA highlighted regional yeast species associations (Figure 3C). Regions had non-intersecting circles suggesting that groups were significantly different. The species that strongly
TABLE 2 | ANOSIM pairwise tests for differences on yeast community composition between regions.

\begin{tabular}{lcccc}
\hline & Santorini & Nemea & Peza & Mantineia \\
\hline Santorini & & $\mathbf{0 . 4 4}$ & $\mathbf{0 . 6 9 5}$ & $\mathbf{0 . 1 0 6}$ \\
Nemea & 0.001 & & $\mathbf{0 . 1 4 9}$ & $\mathbf{0 . 1 4 1}$ \\
Peza & 0.001 & 0.002 & & $\mathbf{0 . 6 2 7}$ \\
Mantineia & 0.11 & 0.1 & 0.001 & \\
\hline
\end{tabular}

Values of $R$ are indicated in bold and values of $p$ in italic. Statistically significant differences are indicated with beige color.

discriminate between regions include $M$. pulcherrima and $S$. bacillaris (strongly associated with Nemea and Peza), A. pullulans (Nemea), H. uvarum (Mantineia), and H. opuntiae and H. guilliermondii (Santorini).

\section{Intra-Regional Yeast Community Similarities}

We also compared the yeast communities within regions, where multiple vineyards were sampled across vintages, i.e., Nemea and Santorini regions (vintages $\mathrm{A}$ and $\mathrm{B}$ ) and Peza region (vintage A). Overall, significant trends of vineyard-specific 


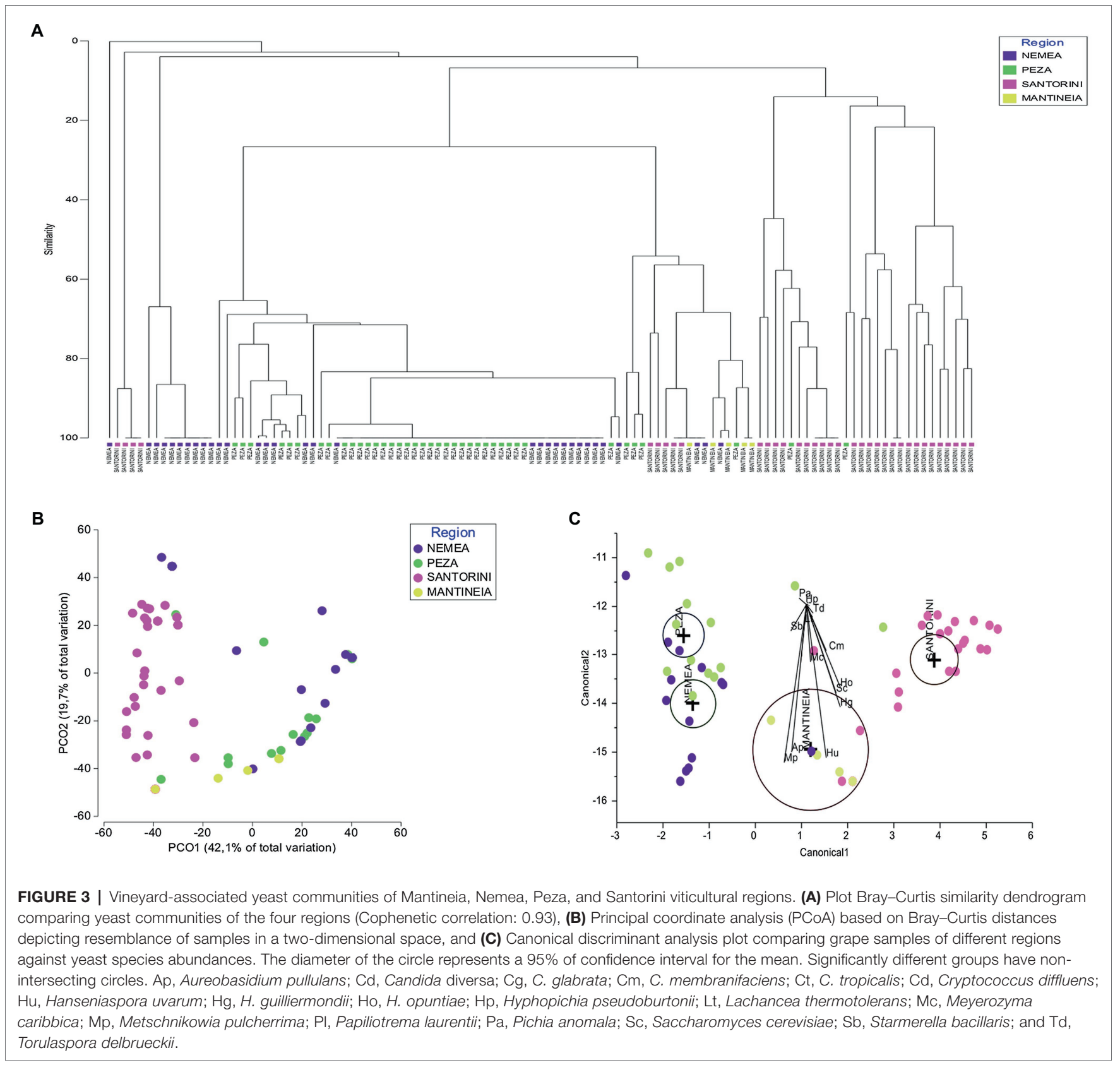

patterns were revealed by both ANOSIM and PERMANOVA tests (ANOSIM: $R=0.508, p=0.001$; PERMANOVA: $R=12.525, p=0.001$; Table 1). Significant dissimilarities were recorded between vineyards in Santorini island, across both vintages $(R=0.471, p=0.001$; Table 1 and Figure 4). Significant differences were also detected between vineyards in Nemea, although dissimilarities were lower in vintage $B$ than A (ANOSIM: $R=0.388, p=0.001$; Table 1 and Figure 4). Although there was not a clear differentiation between Peza vineyards, distinct community patterns were detected between the northern and the southern vineyards. The southern vineyards were characterized by the complete dominance of M. pulcherrima, while the northern vineyards showed much higher biodiversity. By applying nMDS and hierarchical clustering dendrograms grouping of samples according to their vineyard of origin was detected in Peza and Santorini, but it was less obvious in Nemea. We further asked whether there was any structure according to the geographical distance of vineyards but Mantel test did not support such a correlation $(p \geq 0.34)$.

\section{Similarities of Yeasts Communities Over Time}

We examined whether the yeast communities in different viticultural regions are temporarily stable. By applying ANOSIM 

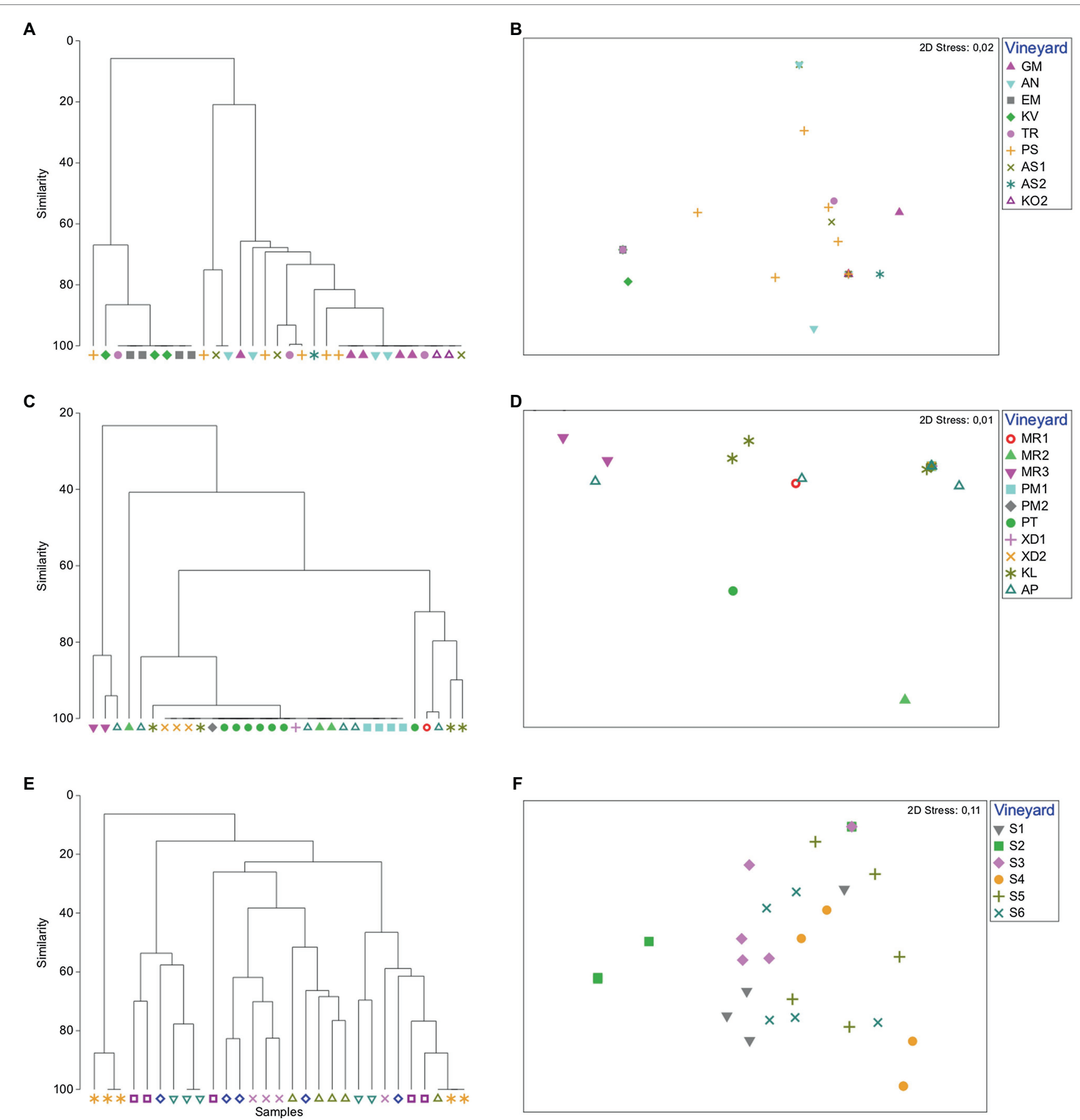

FIGURE 4 | Bray-Curtis non-metric multidimensional scaling plots and dendrograms of yeast community vineyard samples in Nemea (A,B), Peza (C,D), and Santorini $(\mathbf{E}, \mathbf{F})$, respectively.

and PERMANOVA tests, no significant differences were observed over vintages (ANOSIM: $R=0.043, p=0.138$; Table 1). The key yeast species in regional yeast communities were steadily established across vintages in each region, except for Santorini. There was a pronounced differentiation among vintages as per the dominant species in Santorini island, with $H$. opuntiae, H. guilliermondii, and $H$. uvarum prevailing in vintages A, B, and C, respectively. Similar results were obtained with PCoA. Overall, there was not a clear separation of samples from different vintages, suggesting that the yeast communities remained rather unchanged over time (Figure 5A). Only in Santorini, the samples from different vintages were placed separately, particularly those of vintage A (Figure 5B). 

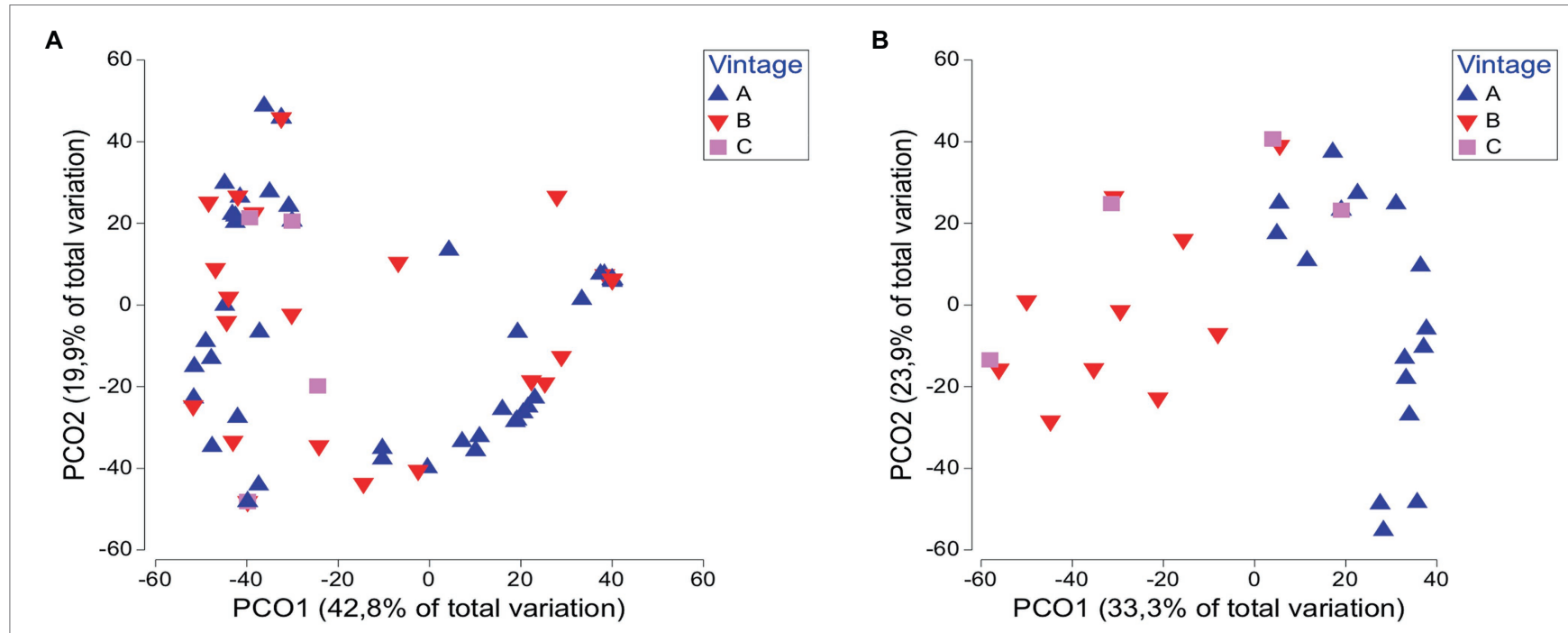

FIGURE 5 | PCoA score plot of yeasts communities across vintages: (A) all regions and (B) Santorini.

\section{Effect of Environmental Conditions}

We further asked whether the environmental conditions (climatic and grape must characteristics) affect regional yeast community delimitation. Significant differences were detected between regions in the majority of the environmental conditions with the exception of the average high and maximum temperatures and the sugar content of grape juice (ANOVA; Supplementary Table S1). Conversely, the environmental conditions did not show statistically significant yearly fluctuations within regions, with a few exceptions (Supplementary Table S2). Environmental factors were moderately related with yeast diversity similarity at Rho $=0.386(p=0.0001$, Spearman rank correlation, 999 permutations).

A BEST was applied to identify the most important environmental factors that drive community differences across regions. BEST showed that among the different environmental factors the maximum temperature could better explain yeast community differentiation (Supplementary Table S3). The maximum temperature along with the elevation was the most highly correlated couple of variables with the yeast community biogeographic patterns (Supplementary Table S3).

According to the distance-based linear model marginal test, there was a significant correlation between yeast assemblages and each of the environmental variables analyzed, except for the sugar content (Supplementary Table S4). Maximum temperature, net precipitation, and elevation were the variables that showed the highest values of $F$, each explaining 19-23\% of the total yeast diversity. Stepwise sequential tests also highlighted the importance of average wind speed and maximum wind speed, which along with average maximum wind speed and average high temperature cumulatively explained approximately 50\% of the variation (Supplementary Table S4). $\mathrm{db}-\mathrm{RDA}$ further justified the variables maximum temperature, net precipitation, elevation, average wind speed, and maximum wind speed as the most critical environmental factors that shape yeast community structures in different viticultural regions.

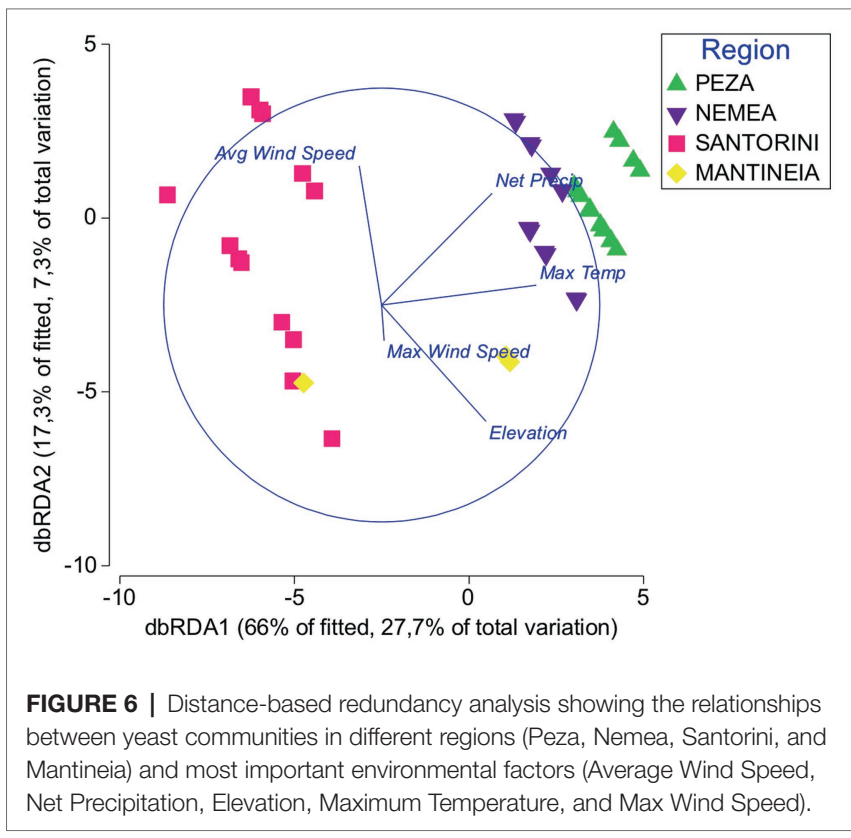

The combination of these factors explained $83.3 \%$ of the total variation (Figure 6).

A partial least squares regression model (PLSR) was applied to demonstrate the association between the set of environmental variables, and the yeast species in order to identify which climatic factors may drive specific yeast populations across regions and vintages. Among the different yeast populations, 11 species explained more than $10 \%$ of the variation in the first two components and were thus retained for the construction of the PLSR model. Projections revealed highly covariable relationships between specific environmental factors and yeast species (Figure 7). In particular, M. pulcherrima was strongly 

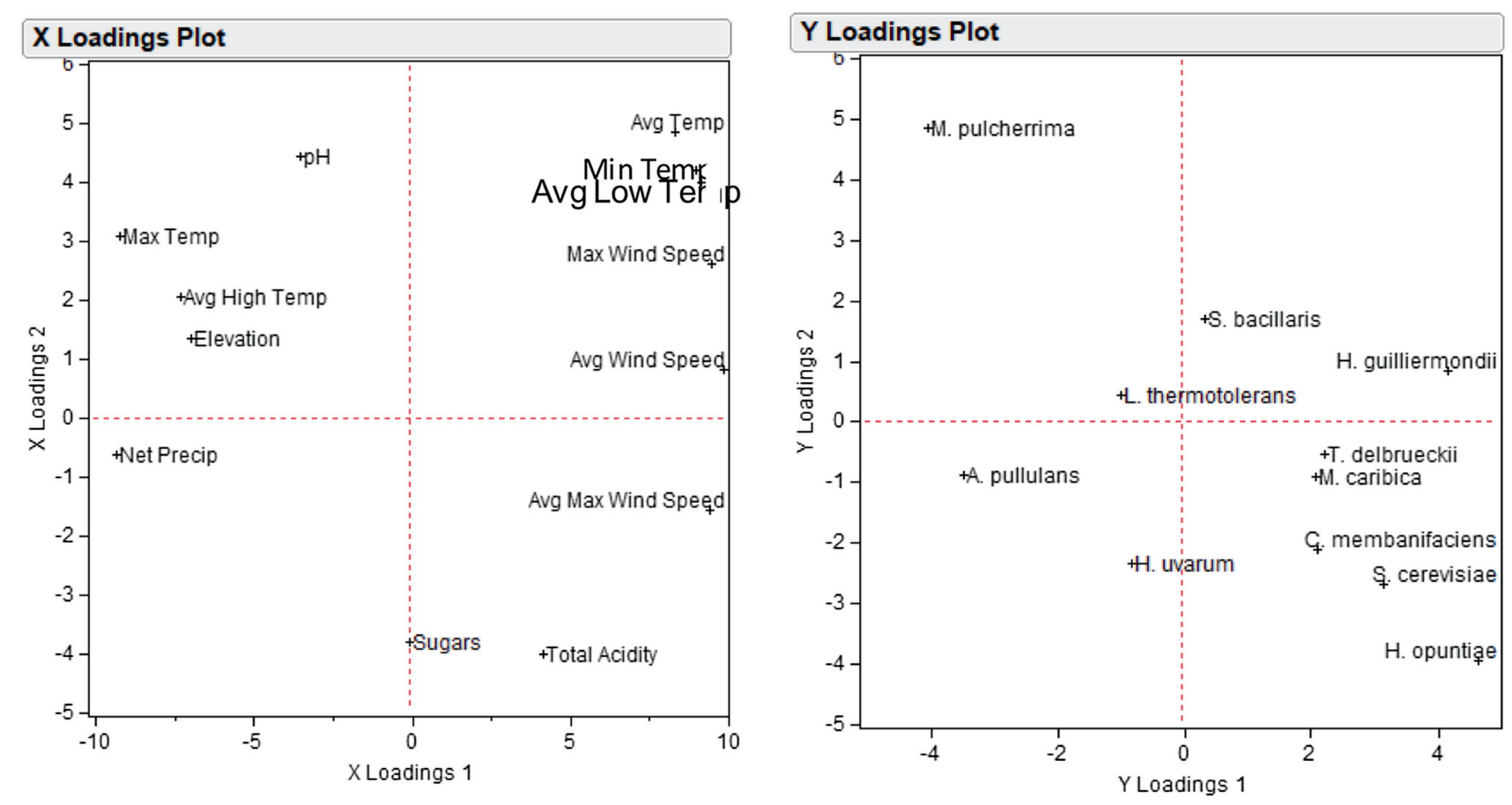

FIGURE 7 | Loading plots of partial least squares regression analysis PLSR of 13 environmental variables (X loadings plot) and 11 yeast populations (Y loadings plot).

associated with $\mathrm{pH}$ and maximum temperature, $H$. guilliermondii with average wind speed, $A$. pullulans with net precipitation, $H$. uvarum with net precipitation and sugars, and $H$. opuntiae with total acidity.

\section{DISCUSSION}

Wine is an important agricultural commodity that exhibits differential regional phenotypes, attributed to the terroir effect. The geographical variation of wines based on their chemical and sensory profiles has been well documented (Fischer et al., 1999; Pereira et al., 2005; Son et al., 2009; López-Rituerto et al., 2012; Robinson et al., 2012). Although differential wine phenotypes among distinct regions establish the terroir hypothesis, the connective causes of the wine terroir concept are still unclear (Van Leeuwen and Seguin, 2006). In this context, the metabolic impact of indigenous yeasts on wine organoleptic features has been documented (Swiegers et al., 2005; Ciani et al., 2010; Nisiotou et al., 2018; Sgouros et al., 2018; Nisiotou et al., 2019). However, whether wine yeast communities are geographically structured causing thereby regional distinctiveness of wines remains to be elucidated.

Here, we surveyed 34 vineyards of four major viticultural zones in Greece. Viticultural zones showed a relatively high inter-regional heterogeneity, compared to analogous studies (Gayevskiy and Goddard, 2012; Drumonde-Neves et al., 2017). This variation can be attributed to the extremely diverse landscape structure, which includes plateau, islands, and hilly regions that, despite their geographic proximity, belong to distinct climatic zones. M. pulcherrima dominated the vineyards of Peza. In Nemea vineyards, M. pulcherrima and A. pullulans were the most common yeasts, while $H$. uvarum dominated Mantineia plateau. Either $H$. guilliermondii or $H$. opuntiae (depending on the vintage) was prevailing Santorini island. Differences were also detected in minor populations and only five out of 18 species were inter-regionally encountered. Notably, several species were region-specific. It is worth mentioning that the same amounts of $H$. uvarum domination (70\%) were previously found in Mantineia plateau (Nisiotou et al., 2007).

Species composition and abundance differed from other wine-producing regions. For instance, a different and more unvarying yeast community composed largely by Rhodotorula glutinis and A. pullulans was observed in three wine-producing regions of New Zealand's vineyards covering about the same area as in the present study (Gayevskiy and Goddard, 2012). H. uvarum, M. pulcherrima, and Pichia terricola were the dominant species in wine-producing regions of the Azores Archipelago of the North Atlantic Ocean (Drumonde-Neves et al., 2017). Several other studies conducted across the world have identified diverse yeast community compositions and abundances. For instance, $H$. uvarum, $M$. pulcherrima, L. thermotolerans, and Rhodotorula sp. were the most frequently isolated yeasts in four South African vineyards (Jolly et al., 2003). H. uvarum, M. pulcherrima, and A. pullulans were the most abundant populations in grapes from 10 vineyards in western Sicily (Settanni et al., 2012). More research aimed at firmly linking a viticultural area with the local yeast community 
will allow identifying global variations and better spot worldwide differences.

At the local scale, each vineyard was found to have a comparatively homogeneous yeast community, which was distinguished from nearby vineyards in the same viticultural region. Differences in the structure of fungal communities between vineyards have been suggested previously (Miura et al., 2017), although the relative information is quite scarce. Grape yeast biota has been also proposed to rely on spatial fluctuation even at the vineyard scale (Setati et al., 2012; Liu et al., 2019). The intra-vineyard fungal diversity has been ascribed to microclimate variability, but there is no experimental validation for this hypothesis. Usually, a vineyard forms a relatively uniform environmental niche as it is subjected to the same pedoclimatic conditions and farming treatments. It is plausible to conclude therefore that, at least for yeast populations, single vineyards may support the growth of a relatively similar species composition (Sabate et al., 2002; Vigentini et al., 2015). We are not certain whether vineyard-associated microbial patterns present a distancedependent model at a local scale. Here, however, it was shown that the geographical distance of vineyards within a region did not correlate with the level of yeast community dissimilarity.

Previous metagenomics studies suggested a non-random distribution of vineyard-associated fungal or bacterial populations, with regional and environmental features being among the most critical factors affecting community assembly (Bokulich et al., 2014; Morrison-Whittle and Goddard, 2018). However, it is not clear whether the above applies to wine yeast populations alone, which constitute a subset of the total fungal community. As a first goal of this study, we conducted multiple grape berry sampling to acquire a comprehensive picture of the spatio-temporal structure of wine yeast communities in the major viticultural zones of Greece. We targeted the culturable wine yeast community rather than fungal populations unrelated to wine production. To this end, it was clearly shown that yeast communities differ significantly at local scale. The differences were ascribed to both the dominant and the minor populations. Viticultural regions were associated with diverse community compositions, which might affect regional characteristics of wines, introducing thereby a microbial notion to wine terroir. Importantly, major players in wine fermentation, like, H. uvarum, $H$. opuntiae, H. guilliermondii, and M. pulcherrima significantly contributed to delimitation among viticultural areas. These yeasts have been shown to confer significant metabolic impact on wine quality (Ciani et al., 2010; Nisiotou et al., 2018, 2019; Sgouros et al., 2018).

To assign a microbial aspect to wine terroir, a temporal stability for regional yeast communities is essential (Chalvantzi et al., 2020). However, the extent of year-to-year fluctuation in the wine yeast community assembly of a viticultural region has hardly been studied. It was previously shown that vintage may significantly affect the vineyard-associated yeast biodiversity (Sabate et al., 2002; Bokulich et al., 2014; Vigentini et al., 2015). As opposed, we did not detect any significant difference between vintages in the different regions surveyed, except for the island of Santorini. This further justifies our conclusion about the distinctiveness of regional wine yeast communities, in that the differences detected are not stochastic but show temporal stability.

The inherent climatic and topographical features of viticultural regions form the basis of the wine terroir. It has been proposed that grapevine microbiomes have a spatial distribution that corresponds to environmental conditions both between and inside vineyards (Griggs et al., 2021). It is unclear, however, which environmental factors affect the grape-associated regional wine yeast communities and explain the magnitude of biodiversity. Here, it was shown that the environmental factors could explain a part of the total yeast biodiversity. BEST-tests revealed maximum temperature, elevation, and net precipitation as the highest correlated variables with the yeast community biogeographic patterns. Similarly, environmental features were previously shown to affect the grape-associated microbial communities, but poor $\mathrm{R}^{2}$ coefficients suggested weak feature importance (Bokulich et al., 2014). Here, distance-based linear model marginal test showed significant correlation between yeast assemblages and each of the environmental variables individually. Maximum temperature, net precipitation, and elevation showed the highest values of F. Factually, elevated altitude forms a hostile environment for microorganisms by exposing cells to ultraviolet (UV) radiation, desiccation, and low temperatures and it was recently shown to significantly affect airborne fungal communities (Tanaka et al., 2019). Net precipitation is another important environmental feature affecting microbial biodiversity as recently shown for soil fungi global niche differentiation (Bahram et al., 2018). We also showed that certain environmental factors may drive the population size of specific yeast populations, like $\mathrm{pH}$ and maximum temperature of $M$. pulcherrima, average wind speed of $H$. guilliermondii, and total acidity of $H$. opuntiae. Net precipitation was found to be highly correlated with A. pullulans and $H$. uvarum. Precipitation levels were previously shown to correlate with the abundance of Metschnikowia, Torulaspora, and Saccharomyces yeast species in grapes from different Chilean valleys (Jara et al., 2016). Given the importance of these species in wine fermentation, it is reasonable to assume that variations in certain climatic conditions can contribute to distinct wine phenotypes by altering specific wine yeast populations.

Our results here support the hypothesis that different regions harbor distinct microbial communities, which was shown to apply specifically to the wine-associated yeast populations. We also highlight the implication of climatic features in regional wine yeast biogeography. The fact, however, that major environmental features, like climatic and grape must characteristics, could explain only a part of biogeography points to the complexity of the factors driving wine yeast community composition. Other regional factors, including modes of yeast dispersion parameters, landscape composition or biotic interactions may also interfere in community assembly.

\section{DATA AVAILABILITY STATEMENT}

The original contributions presented in the study are included in the article/Supplementary Material, and further inquiries can be directed to the corresponding author. 


\section{AUTHOR CONTRIBUTIONS}

AN designed the study, performed experiments, analyzed data, and wrote the paper. IC conducted experiments and analyzed data. CT contributed to data analysis and writing the paper. GB analyzed data and contributed to writing the paper. All authors contributed to the article and approved the submitted version.

\section{FUNDING}

This research has been partially supported by the European Union and Greek national funds through the Operational Program Competitiveness, Entrepreneurship and Innovation,

\section{REFERENCES}

Alexandre, H. (2020). Wine yeast terroir: separating the wheat from the chafffor an open debate. Microorganisms 8:787. doi: 10.3390/microorganisms8050787 Anderson, M. J., Gorley, R. N., and Clarke, K. R. (2008). PERMANOVA+ for PRIMER: Guide to Software and Statistical Methods. Plymouth, UK: PRIMER-E.

Anderson, M. J., and Willis, T. J. (2003). Canonical analysis of principal coordinates: a useful method of constrained ordination for ecology. Ecology 84, 511-525. doi: 10.1890/0012-9658(2003)084[0511:CAOPCA]2.0.CO;2

Bahram, M., Hildebrand, F., Forslund, S. K., Anderson, J. L., Soudzilovskaia, N. A., Bodegom, P. M., et al. (2018). Structure and function of the global topsoil microbiome. Nature 560, 233-237. doi: 10.1038/s41586-018-0386-6

Banilas, G., Sgouros, G., and Nisiotou, A. (2016). Development of microsatellite markers for Lachancea thermotolerans typing and population structure of wineassociated isolates. Microbiol. Res. 193, 1-10. doi: 10.1016/j.micres.2016.08.010

Barata, A., Malfeito-Ferreira, M., and Loureiro, V. (2012). The microbial ecology of wine grape berries. Int. J. Food Microbiol. 153, 243-259. doi: 10.1016/j. ijfoodmicro.2011.11.025

Bokulich, N. A., Collins, T. S., Masarweh, C., Allen, G., Heymann, H., Ebeler, S. E., et al. (2016). Associations among wine grape microbiome, metabolome, and fermentation behavior suggest microbial contribution to regional wine characteristics. mBio 7, 1-12. doi: 10.1128/mBio.00631-16

Bokulich, N. A., Thorngate, J. H., Richardson, P. M., and Mills, D. A. (2014). Microbial biogeography of wine grapes is conditioned by cultivar, vintage, and climate. Proc. Natl. Acad. Sci. U. S. A. 111, E139-E148. doi: 10.1073/ pnas. 1317377110

Chalvantzi, I., Banilas, G., Tassou, C., and Nisiotou, A. (2020). Patterns of genetic diversity and the invasion of commercial starters in Saccharomyces cerevisiae vineyard populations of Santorini island. Foods 9, 6-8. doi: 10.3390/ foods 9050561

Chavan, P., Mane, S., Kulkarni, G., Shaikh, S., and Ghormade, V. (2009). Natural yeast flora of different varieties of grapes used for wine making in India. Food Microbiol. 26, 801-808. doi:10.1016/j.fm.2009.05.005

Ciani, M., Comitini, F., Mannazzu, I., and Domizio, P. (2010). Controlled mixed culture fermentation: a new perspective on the use of non-Saccharomyces yeasts in winemaking. FEMS Yeast Res. 10, 123-133. doi: 10.1111/j.1567-1364.2009.00579.x

Clarke, K. R., and Gorley, R. N. (2015). PRIMER v7: User Manual/Tutorial. Plymouth, UK: PRIMER-E.

Cline, L. C., and Zak, D. R. (2014). Dispersal limitation structures fungal community assembly in a long-term glacial chronosequence. Environ. Microbiol. 16, 1538-1548. doi: 10.1111/1462-2920.12281

Cordero-Bueso, G., Arroyo, T., Serrano, A., Tello, J., Aporta, I., Vélez, M. D., et al. (2011). Influence of the farming system and vine variety on yeast communities associated with grape berries. Int. J. Food Microbiol. 145, 132-139. doi: 10.1016/j.ijfoodmicro.2010.11.040

Drumonde-Neves, J., Franco-Duarte, R., Lima, T., Schuller, D., and Pais, C. (2017). Association between grape yeast communities and the vineyard ecosystems. PLoS One 12:e169883. doi: 10.1371/journal.pone.0169883 under the call RESEARCH - CREATE - INNOVATE (project code: T1EDK - 04747; MIS 5031228).

\section{ACKNOWLEDGMENTS}

We would like to thank Dimitra Dourou and Maria-Evangelia Filippousi for their help during the experiments and technical assistance.

\section{SUPPLEMENTARY MATERIAL}

The Supplementary Material for this article can be found online at https://www.frontiersin.org/articles/10.3389/fmicb.2021.705001/ full\#supplementary-material

Esteve-Zarzoso, B., Belloch, C., Uruburu, F., and Querol, A. (1999). Identification of yeasts by RFLP analysis of the 5.8S rRNA gene and the two ribosomal internal transcribed spacers. Int. J. Syst. Bacteriol. 49, 329-337. doi: 10.1099/00207713-49-1-329

Finkel, O. M., Burch, A. Y., Elad, T., Huse, S. M., Lindow, S. E., Post, A. F., et al. (2012). Distance-decay relationships partially determine diversity patterns of phyllosphere bacteria on Tamrix trees across the Sonoran Desert. Appl. Environ. Microbiol. 78, 6187-6193. doi: 10.1128/AEM.00888-12

Fischer, U., Roth, D., and Christmann, M. (1999). The impact of geographic origin, vintage and wine estate on sensory properties of Vitis vinifera cv. Riesling wines. Food Qual. Prefer. 10, 281-288. doi: 10.1016/S0950-3293 (99)00008-7

Gayevskiy, V., and Goddard, M. R. (2012). Geographic delineations of yeast communities and populations associated with vines and wines in New Zealand. ISME J. 6, 1281-1290. doi: 10.1038/ismej.2011.195

Griggs, R. G., Steenwerth, K. L., Mills, D. A., and Bokulich, N. A. (2021). Sources and assembly of microbial communities in vineyards as a functional component of winegrowing. Front. Microbiol. 12:673810. doi: 10.3389/ fmicb.2021.673810

Jara, C., Laurie, V. F., Mas, A., Romero, J., Università, S., and Romero, J. (2016). Microbial terroir in Chilean valleys: diversity of non-conventional yeast. Front. Microbiol. 7:663. doi: 10.3389/fmicb.2016.00663

Jolly, N. P., Augustyn, O. P. H., and Pretorius, I. S. (2003). The occurrence of non-Saccharomyces cerevisiae yeast species over three vintages in four vineyards and grape musts from four production regions of the Western cape, South Africa. S. Afr. J. Enol. Vitic. 24, 8-10. doi: 10.21548/24-2-2640

Knight, S., Klaere, S., Fedrizzi, B., and Goddard, M. R. (2015). Regional microbial signatures positively correlate with differential wine phenotypes: evidence for a microbial aspect to terroir. Sci. Rep. 5:14233. doi: 10.1038/ srep 14233

Legendre, P., and Legendre, L. (1998). Numerical Ecology. Amsterdam: Elsevier Science BV.

Li, S. S., Chen, C., Li, Z., Chen, J. Y., Yan, B., Han, B. Z., et al. (2010). Yeast species associated with wine grapes in China. Int. J. Food Microbiol. 138, 85-90. doi: 10.1016/j.ijfoodmicro.2010.01.009

Liu, D., Zhang, P., Chen, D., and Howell, K. (2019). From the vineyard to the winery: how microbial ecology drives regional distinctiveness of wine. Front. Microbiol. 10:2679. doi: 10.3389/fmicb.2019.02679

López-Rituerto, E., Savorani, F., Avenoza, A., Busto, J. H., Peregrina, J. M., and Engelsen, S. B. (2012). Investigations of la Rioja terroir for wine production using 1H NMR metabolomics. J. Agric. Food Chem. 60, 3452-3461. doi: $10.1021 /$ jf204361d

Miura, T., Sánchez, R., Castañeda, L. E., Godoy, K., and Barbosa, O. (2017). Is microbial terroir related to geographic distance between vineyards? Environ Microbiol. Rep. 9, 742-749. doi: 10.1111/1758-2229.12589

Morrison-Whittle, P., and Goddard, M. R. (2015). Quantifying the relative roles of selective and neutral processes in defining eukaryotic microbial communities. ISME J. 9, 2003-2011. doi: 10.1038/ismej.2015.18 
Morrison-Whittle, P., and Goddard, M. R. (2018). From vineyard to winery: a source map of microbial diversity driving wine fermentation. Environ. Microbiol. 20, 75-84. doi: 10.1111/1462-2920.13960

Nisiotou, A. A., and Nychas, G. J. E. (2007). Yeast populations residing on healthy or botrytis-infected grapes from a vineyard in Greece. Appl. Environ. Microbiol. 73, 2765-2768. doi: 10.1128/AEM.01864-06

Nisiotou, A. A., Spiropoulos, A. E., and Nychas, G. J. E. (2007). Yeast community structures and dynamics in healthy and botrytis-affected grape must fermentations. Appl. Environ. Microbiol. 73, 6705-6713. doi: 10.1128/AEM.01279-07

Nisiotou, A., Mallouchos, A., Tassou, C., and Banilas, G. (2019). Indigenous yeast interactions in dual-starter fermentations may improve the varietal expression of Moschofilero wine. Front. Microbiol. 10:1712. doi: 10.3389/ fmicb.2019.01712

Nisiotou, A., Sgouros, G., Mallouchos, A., Nisiotis, C. S., Michaelidis, C., Tassou, C., et al. (2018). The use of indigenous Saccharomyces cerevisiae and Starmerella bacillaris strains as a tool to create chemical complexity in local wines. Food Res. Int. 111, 498-508. doi: 10.1016/j.foodres.2018.05.035

OIV (2015). Compendium of international methods of wine and must analysis. Paris: France International Organisation of Vine and Wine (OIV).

Peay, K. G., Garbelotto, M., and Bruns, T. D. (2010). Evidence of dispersal limitation in soil microorganisms: isolation reduces species richness on mycorrhizal tree islands. Ecology 91, 3631-3640. doi: 10.1890/09-2237.1

Pereira, G. E., Gaudillere, J. P., Van Leeuwen, C., Hilbert, G., Lavialle, O., Maucourt, M., et al. (2005). 1H NMR and chemometrics to characterize mature grape berries in four wine-growing areas in France. J. Agric. Food Chem. 53, 6382-6389. doi: 10.1021/jf058058q

Pinto, C., Pinho, D., Cardoso, R., Custódio, V., Fernandes, J., Sousa, S., et al. (2015). Wine fermentation microbiome: a landscape from different Portuguese wine appellations. Front. Microbiol. 6:905. doi: 10.3389/fmicb.2015.00905

Ramette, A. (2007). Multivariate analyses in microbial ecology. FEMS Microbiol. Ecol. 62, 142-160. doi: 10.1111/j.1574-6941.2007.00375.x

Robinson, A. L., Adams, D. O., Boss, P. K., Heymann, H., Solomon, P. S., and Trengove, R. D. (2012). Influence of geographic origin on the sensory characteristics and wine composition of Vitis vinifera $\mathrm{cv}$. Cabernet sauvigon wines from Australia. Am. J. Enol. Vitic. 63, 467-476. doi: 10.5344/ajev.2012.12023

Sabate, J., Cano, J., Esteve-Zarzoso, B., and Guillamon, J.-M. (2002). Isolation and identification of yeasts associated with vineyard and winery by RFLP analysis of ribosomal genes and mitochondrial DNA. Microbiol. Res. 157, 267-274. doi: 10.1078/0944-5013-00163

Sawatsky, M., Clyde, M., and Meek, F. (2015). Partial least squares regression in the social sciences. Quant. Methods Psychol. 11, 52-62. doi: 10.20982/ tqmp.11.2.p052

Setati, M. E., Jacobson, D., Andong, U., and Bauer, F. (2012). The vineyard yeast microbiome, a mixed model microbial map. PLoS One 7:e52609. doi: 10.1371/journal.pone.0052609
Settanni, L., Sannino, C., Francesca, N., Guarcello, R., and Moschetti, G. (2012). Yeast ecology of vineyards within Marsala wine area (western Sicily) in two consecutive vintages and selection of autochthonous Saccharomyces cerevisiae strains. J. Biosci. Bioeng. 114, 606-614. doi: 10.1016/j. jbiosc.2012.07.010

Sgouros, G., Chalvantzi, I., Mallouchos, A., Paraskevopoulos, Y., Banilas, G., and Nisiotou, A. (2018). Biodiversity and enological potential of nonSaccharomyces yeasts from Nemean vineyards. Fermentation 4:32. doi: 10.3390/ fermentation 4020032

Son, H. S., Hwang, G. S., Kim, K. M., Ahn, H. J., Park, W. M., Van Den Berg, F., et al. (2009). Metabolomic studies on geographical grapes and their wines using $1 \mathrm{H}$ NMR analysis coupled with multivariate statistics. J. Agric. Food Chem. 57, 1481-1490. doi: 10.1021/jf803388w

Swiegers, J. H., Bartowsky, E. J., Henschke, P. A., and Pretorius, I. S. (2005). Yeast and bacterial modulation of wine aroma and flavour. Aust. J. Grape Wine Res. 11, 139-173. doi: 10.1111/j.1755-0238.2005.tb00285.x

Tanaka, D., Sato, K., Goto, M., Fujiyoshi, S., Maruyama, F., Takato, S., et al. (2019). Airborne microbial communities at high-altitude and suburban sites in Japan suggest a new perspective for bioprospecting. Front. Bioeng. Biotechnol. 7:12. doi: 10.3389/fbioe.2019.00012

Taylor, M. W., Tsai, P., Anfang, N., Ross, H. A., and Goddard, M. R. (2014). Pyrosequencing reveals regional differences in fruit-associated fungal communities. Environ. Microbiol. 16, 2848-2858. doi: 10.1111/1462-2920.12456

Van Leeuwen, C., and Seguin, G. (2006). The concept of terroir in viticulture. J. Wine Res. 17, 1-10. doi: 10.1080/09571260600633135

Vigentini, I., De Lorenzis, G., Fabrizio, V., Valdetara, F., Faccincani, M., Panont, C. A., et al. (2015). The vintage effect overcomes the terroir effect: a three year survey on the wine yeast biodiversity in Franciacorta and Oltrepò Pavese, two northern Italian vine-growing areas. Microbiology 161, 362-373. doi: 10.1099/mic.0.000004

White, T. J., Bruns, T., Lee, S., and Taylor, J. (1990). Amplification and direct sequencing of fungal ribosomal RNA genes for phylogenetics. PCR Protoc. 1990, 315-322. doi: 10.1016/b978-0-12-372180-8.50042-1

Conflict of Interest: The authors declare that the research was conducted in the absence of any commercial or financial relationships that could be construed as a potential conflict of interest.

Copyright (C) 2021 Chalvantzi, Banilas, Tassou and Nisiotou. This is an open-access article distributed under the terms of the Creative Commons Attribution License (CC BY). The use, distribution or reproduction in other forums is permitted, provided the original author(s) and the copyright owner(s) are credited and that the original publication in this journal is cited, in accordance with accepted academic practice. No use, distribution or reproduction is permitted which does not comply with these terms. 